\title{
Thyroglossal duct cyst: dynamic ultrasound evaluation and sonoanatomy revisited
}

\author{
Ke-Vin Chang ${ }^{1}$, Wei-Ting Wu ${ }^{1}$, Levent Özçakar ${ }^{2}$
}

${ }^{1}$ Department of Physical Medicine and Rehabilitation, National Taiwan University Hospital, BeiHu Branch and National Taiwan University College of Medicine, Taipei, Taiwan, ${ }^{2}$ Department of Physical and Rehabilitation Medicine, Hacettepe University Medical School, Ankara, Turkey

\section{To the Editor,}

A 4-year-old boy had a neck tumor excised six months ago. The pathology had been reported as an epitheliumlined mucus-filed cyst. He was sent for an ultrasound (US) examination as part of the routine post-operative check-up although his parents did not find any swelling on his neck and physical examination revealed no palpable lump. Initial US imaging showed no mass at the anterior upper half of his neck. However, while sweeping the transducer across the head and neck junction, a compressible cyst-like lesion was seen, gliding in accordance with swallowing (fig 1, Video 1, on the journal site). A tract was also noticed at the bottom of the cyst, piercing the muscle of the tongue base. Under the impression of a recurrent thyroglossal ductal cyst, he was referred back to the surgical department.

The thyroglossal ductal cyst (TGDC) is the most common congenital cystic lesion in the neck, whereby its prevalence is reported to be $7 \%$ in the general population [1]. The mass is usually asymptomatic and develops near the midline. Approximately $60 \%$ of the TGDCs are located between the thyroid gland and the hyoid bone, while the remaining $40 \%$ are found in the suprahyoid, suprasternal and intra-lingual regions. The TGDC may be complicated with infection or a transformation to carcinomas [2]. Therefore, prompt recognition by using imaging modalities such as US and computed tomography is of paramount clinical importance.

Received 08.01.2019 Accepted 20.01.2019

Med Ultrason

2019, Vol. 21, No 1, 99-100, DOI: 10.11152/mu-1879

Corresponding author: Ke-Vin Chang, $\mathrm{MD}, \mathrm{PhD}$

Department of Physical Medicine and Rehabilitation, National Taiwan University Hospital Bei-Hu Branch and National Taiwan University College of Medicine, Taipei, Taiwan E-mail: kvchang011@gmail.com
The usefulness of US imaging as a follow-up tool after a neck mass excision is demonstrated in the present case. The TGDC appeared as a well-demarcated anechoic cystic lesion during the US assessment [3]. Posterior enhancement is usually seen as the cysts are filled with mucus. Visualization of the ductal compartment is helpful for the diagnosis but may also be difficult due to its deeper location. In this sense, the transducer is suggested to be localized against the chin in the sagittal plane approximating the hyoid bone on its one end. At least three muscle layers can be appreciated: the mylohyoid, geniohyoid and genioglossus muscles. The anterior belly of

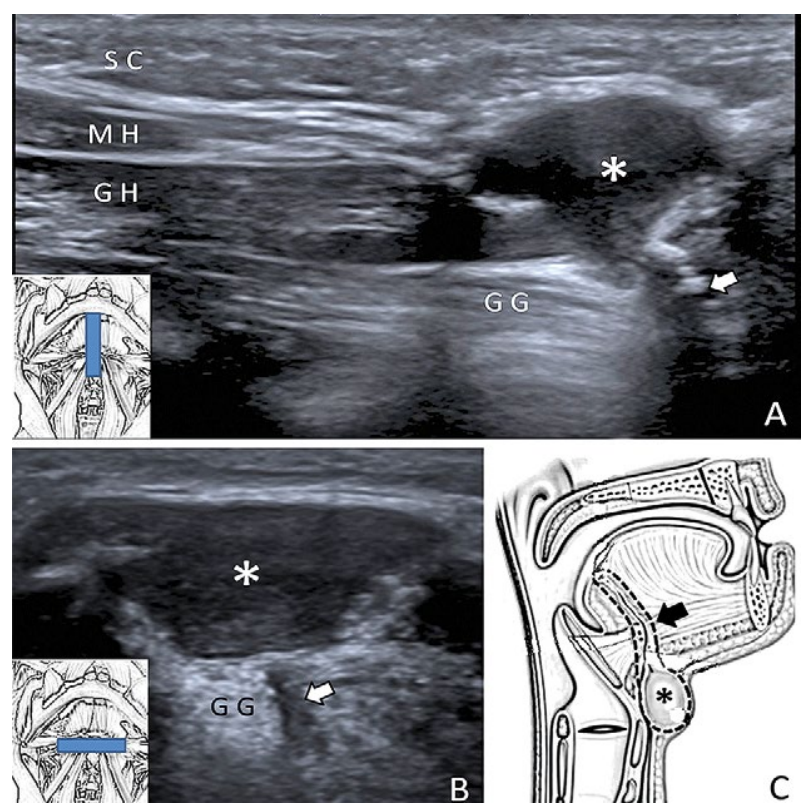

Fig 1. Sagittal (A) and transverse (B) ultrasound imaging and schematic drawing (C) of a recurrent thyroglossal duct cyst (*). Arrows indicate the ductal portion of the cyst. $\mathrm{MH}$, mylohyoid muscle; GH, geniohyoid muscle; GG, genioglossus muscle; $\mathrm{SC}$, subcutaneous fat. 
the digastric muscle may be seen at the mandibular end when the transducer is positioned right on the midline. The duct can be found located anterior to the hyoid bone, piercing the suprahyoid and glossal muscles.

Because adhesion with the hyoid bone is common in TGDCs, synchronous movements of the TGDC and the hyoid bone can be used as a diagnostic clue during dynamic US assessment. Herein, another common cystic lesion that should be differentiated from the TGDC is the epidermoid cyst. Lack of concomitant gliding with the hyoid bone, absence of a duct connecting the oral cavity and the presence of more echogenic dots over the hypoechoic background would be the hallmarks of an epidermoid cyst [4]. Of note, in cases where these cysts become infected, the echogenic dots might be present in both. Last but not least, recurrence of TGDCs is not uncommon, even using the Sistrunk procedure (removal of the cyst with partial excision of the hyoid bone) [2]. The present case also highlights the importance of routine/ convenient US follow-ups after surgery.
Acknowledgment: The present study is supported by the National Taiwan University Hospital, Bei-Hu Branch, Ministry of Science and Technology (MOST 106-2314-B-002-180-MY3) and Taiwan Society of U1trasound in Medicine.

\section{References}

1. Mondin V, Ferlito A, Muzzi E, et al. Thyroglossal duct cyst: personal experience and literature review. Auris Nasus Larynx 2008;35:11-25.

2. Chou J, Walters A, Hage R, et al. Thyroglossal duct cysts: anatomy, embryology and treatment. Surg Radiol Anat 2013;35:875-881.

3. Bansal AG, Oudsema R, Masseaux JA, Rosenberg HK. US of Pediatric Superficial Masses of the Head and Neck. Radiographics 2018;38:1239-1263.

4. Choi HI, Choi YH, Cheon JE, Kim WS, Kim IO. Ultrasonographic features differentiating thyroglossal duct cysts from dermoid cysts. Ultrasonography 2018;37:71-77. 